ISAHP 1996, Vancouver, Canada, July 12-15, 1996

\title{
EXPERIMENTS WITH A NEURAL NETWORK APPROACH TO AHP WITH INTERVAL JUDGEMENTS
}

\author{
Paul Schreuder, Doncho Petkov and Linda Haines \\ Faculty of Science \\ University of Natal,Private bag X01, Pietermaritzburg 3209, South Africa \\ petkov@comp.unp.ac.za/ hainesl@stats.unp.ac.za
}

\begin{abstract}
Existing methods for determining preference assessments from interval judgements are a simulation approach (Saaty and Vargas, 1987), a linear programming approach (Arbel , 1989), (Salo and Hamalainen, 1991) and a neural network approach (Hao and Vargas, 1991). The simulation approach on its own is time consuming and hence is not a computationally viable solution while the Hao and Vargas neural network technique requires further research in order to determine its suitability. The only technique which has thus far been practically implemented is the linear programming technique by Salo and Hamalainen in the software package INPRE .
\end{abstract}

This research presents an approach which encapsulates the strengths of simulation and the generalizing and function approximation capabilities of neural networks within given computational and time constraints. The difference from the Saaty and Vargas method is that simulation is performed on a log scale over the intervals, so that all inconsistent matrices possible from the interval judgement matrix are equally likely. The proposed network is a two layered network which is fully connected and uses the back propagation algorithm as the learning algorithm.

An experiment was carried to examine the effect that the independent variables ,average consistency ratio of training set and average consistency ratio of test set, have on the dependant variable, validation error. The hypothesis was that input sets with an average consistency ratio near zero will perform well for test sets with average consistency ratios near zero and performance will decrease as the average consistency ratio increases for both sets.

It can be concluded also that there is a complex relationship between the consistency in the input data and the performance of the trained network. Training sets with low average consistency ratios only perform well with similar test sets. Training sets with large average consistency ratios do not perform well for test sets with low average consistency ratios, but perform acceptably for tests set with high average consistency ratios. This indicates that the network is starting to overcome the inconsistency in the training set which could be thought of as "noise" in the input data.

Saaty recommends that a consistency ratio greater than 0.1 indicates a need to reassess the comparisons. At the same time, it is also realistic to assume that most judgement matrices will have a certain degree of inconsistency. It was interesting to observe that the network trained by training sets with consistency ratios from 0.05 to 0.1 performed better than those with higher consistency ratios and a training set with average consistency ratio of 0.0889 performed in the best way over all test sets. Thus Saaty's guideline determining the upper acceptable bound of the inconsistency ratio to be 0.1 was proven experimentally by this experiment.

Possible areas for further research are the effects of interval size on network performance and the use of refinements to the backpropagation algorithm for training of the network or exploring ergonomic issues of the human-computer interface for software using a neural network approach towards AHP with interval judgements.

\section{References:}

Arbel, A , (1989), Approximate Articulation of Preference and Priority Derivation,

Eur. J. Oper. Res. , No 43, pp317-326.

Hao, G , Vargas, L.G , (1991) Using Neural Networks for Priority Derivation with Interval Judgments ,Proceeding of the MCDM Conference.

Saaty, T.L , Vargas, L.G, (1987) Uncertainty and Rank Order in the Analytical Hierarchy Process, Eur. J. Oper. Res. , No 2, pp. 107-117.

Salo, A.A, Hamalainen, R.P , (1991) Interactive Decision Support through Interval

Judgements , Proceeding of the MCDM Conference in Washington D.C., August 1990. 